\title{
Benefit of an electronic head-mounted Low Vision Aid
}

3

4

Michael D. Crossland ${ }^{1}$, Sandra D. Starke ${ }^{2,3}$, Piotr Imielski ${ }^{3}$, James S. Wolffsohn ${ }^{4}$, Andrew R. Webster ${ }^{1}$ 5

6

${ }^{1} \mathrm{NIHR}$ Biomedical Research Centre for Ophthalmology at Moorfields Eye Hospital and UCL Institute

7

8

9

10

11

12

13

14

15

16

17

18

19

20

21

22

23

24

25
Word count: 4293

Number of tables: 2

Number of figures: 3

\section{Correspondence to:}

Michael Crossland

Department of Optometry

Moorfields Eye Hospital NHS Foundation Trust

162 City Road

London

EC1V 2PD

UK

Email: mcrossland@nhs.net 
27 SDS and PI received salary support from Vision Technologies Ltd during this study. MDC, JSW and

28 ARW have no conflict of interest and have no proprietary interest in any of the materials mentioned

29 in this article.

30

\section{FUNDING STATEMENT}

32 This work was supported by the Technology Strategy Board (InnovateUK) (Biomedical Catalyst 2017

33 Round 1 - Primer (Digital Health Catalyst)). The views expressed are those of the authors and not

34 necessarily those of Moorfields Eye Hospital, the National Institute for Health Research or the

35 Department of Health and Social Care.

36

37 
Purpose: To evaluate the efficacy of electronic head-mounted Low Vision Aid (ehLVA) SightPlus

(GiveVision, UK) and to determine which people with low vision would see themselves likely using an ehLVA like this.

Methods: Sixty participants with Low Vision aged 18 to 93 used SightPlus during an in-clinic study session using a mixed methods design. Visual acuity (ETDRS), contrast sensitivity (Pelli Robson) and reading performance (MNREAD) were measured binocularly at baseline (no device), with the device in 'normal' mode (zoom only), and with preferred enhanced mode (zoom and one out of four digital image enhancements). At the end of the session, a short questionnaire recorded willingness to use an ehLVA like SightPlus, potential use cases, positive/negative comments and adverse effects.

Results: Binocular distance visual acuity improved significantly by $0.63 \log M A R$ on average $(p<0.0001)$ to $0.20 \log$ MAR. Contrast sensitivity improved significantly by $0.22 \log$ units $(p<0.0001)$ to 1.21 log units with zoom only and by $0.40 \log$ units to $1.37 \log$ units with zoom and preferred image enhancement. Reading performance improved significantly for near visual acuity and critical print size $(p<0.015)$, however reading speed significantly decreased $(p<0.0001)$. Nearly half $(47 \%)$ of the participants indicated they would use an ehLVA like SightPlus, especially for television, reading and entertainment (e.g. theatre). Multivariate logistic regression showed that proportion of lifetime affected, baseline contrast sensitivity and use of electronic LVAs explained $41 \%$ of the variation in willingness to use.

Conclusions: SightPlus improves visual function in people with low vision and would be used in its current form by half of the people who tried it. Adverse effects were rare and resolved when the device was removed. Future work should focus on comparing ehLVAs through repeatable real-world tasks and impact on quality of life. 
Electronic head-mounted Low Vision Aids (ehLVAs) have experienced a technological step change over the last decade. ${ }^{12}$ A 2017 review of head-mounted displays for people with low vision highlighted the advantages of such technology over conventional desk-mounted or handheld sight aids. ${ }^{3}$ Since early commercial devices such as the Low Vision Enhancement System were first produced in the $1990 \mathrm{~s},{ }^{4}$ advances in consumer technology have led to the availability of smaller, lighter, more versatile ehLVAs, including SightPlus (GiveVision, Birmingham, UK), eSight (eSight Corporation, Toronto, ON, Canada), IrisVision (IrisVision Global, Pleasanton, CA, USA), OxSight (OxSight Ltd, Oxford, UK) the new JORDY (Enhanced Vision Services, Huntington Beach, CA, USA), with several other devices being developed. ${ }^{5-8}$ SightPlus is a commercially available ehLVA and registered as a Class I medical device. It is designed around a smartphone (serving as a camera, image processor and display) inserted into a virtual reality (VR) headset serving as the optical system. To date, clinical evidence for the efficacy of VR-and-smartphone-based ehLVAs such as SightPlus is lacking.

Modern ehLVAs can embed digital sight enhancement algorithms that have been proven effective in laboratory settings for decades. ${ }^{9-16}$ A 2015 review of 37 research papers highlighted the benefit of various image processing techniques for the visually impaired, albeit with inconsistent preferences between people. ${ }^{1} \mathrm{~A}$ more recent review of ehLVAs illustrated existing strategies to sight enhancement and showed improvements in contrast sensitivity. ${ }^{2}$ However, to date, only two studies have been published demonstrating efficacy of wearables across substantial patient numbers: one for the eSight ehLVA device, ${ }^{17}$ and one for Orcam, a spectacle mounted text-to-speech system. ${ }^{18}$ The eSight study showed improvements in visual acuity, contrast sensitivity, reading performance and functional vision in a self-selected group of 51 adults who had demonstrated motivation to wear the device. Data are lacking for people unaware of ehLVAs or for a more general population of people with visual impairment. 
90 In this study, we evaluated the performance of SightPlus (GiveVision, Birmingham, UK; Figure 1).

91 This device was chosen as it was commercially available in the United Kingdom, was relatively

92 affordable, and there were anecdotal reports of significant visual benefits associated with this

93 device. We hypothesised that acuity, contrast sensitivity and reading speed would improve similarly

94 to a different ehLVA using different technology (eSight). We anticipated that poorer baseline acuity

95 and familiarity with electronic low vision aids would predict willingness to use the device.

96

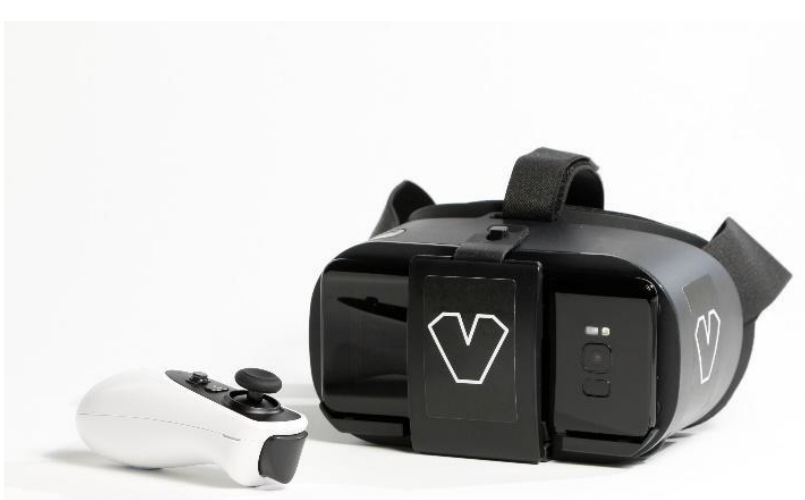

97

98

Figure 1. The SightPlus device, with its wireless remote control. 


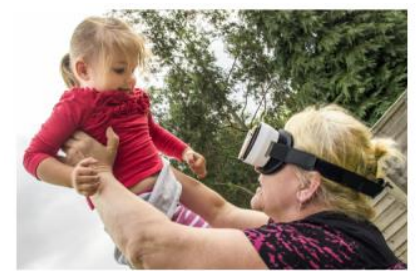

Benefit of a head-mounted augmented reality sight enhancement aid for people with low vision.

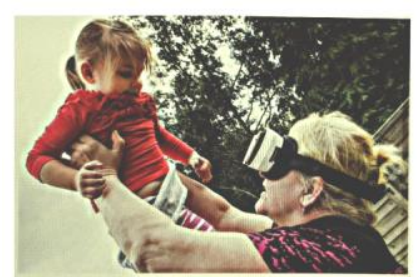

Benefit of a head-mounted augmented reality sight enhancement aid for people with low vision.

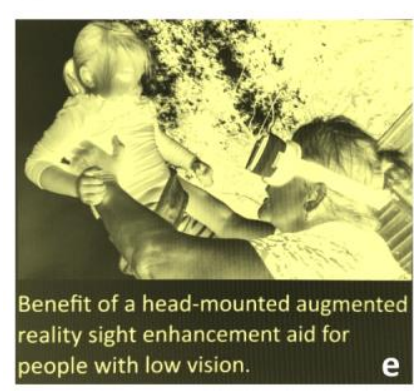

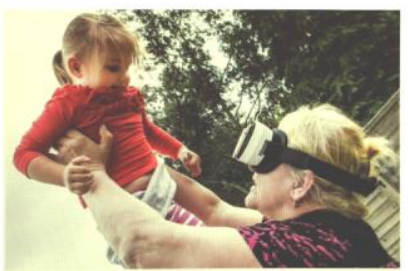

Benefit of a head-mounted augmented reality sight enhancement aid for people with low vision.

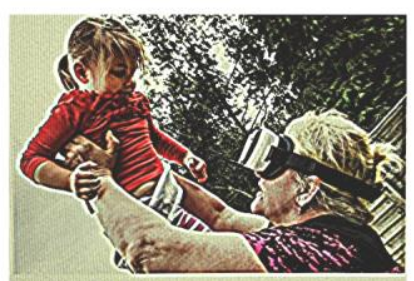

Benefit of a head-mounted augmented reality sight enhancement aid for people with lowvision. d

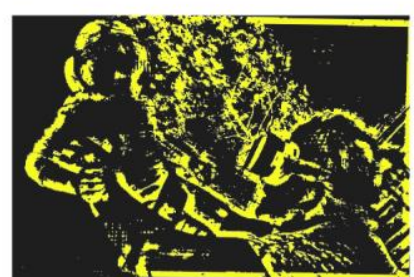

Benefit of a head-mounted augmente reality sight enhancement aid for people with low vision.

Figure 2. Text and a pictorial scene as displayed through five different modes offered by SightPlus. mode.

Materials and Methods

\section{Ethics}

Ethical approval for the study was obtained from the Health Research Authority (IRAS number

237229), and local approval was obtained from the North Thames Clinical Research Network.

Participants gave written informed consent after the purpose of the study was explained and prior to any data collection. 

Hospital. This clinic is in a tertiary centre with a relatively high proportion of younger patients with inherited retinal disease.$^{19}$ All participants met the definition of visual impairment suggested by Leat: having best monocular or binocular visual acuity of worse than $6 / 7.5$, horizontal visual field of less than 146 degrees to Goldmann III4e targets, or contrast sensitivity worse than 1.5 log units. ${ }^{20}$ All were over 18 years of age, fluent in written and spoken English and able to hear and understand instructions whilst wearing the device.

Materials

SightPlus offers proprietary sight enhancement software through a smartphone (S8, Samsung, South Korea) mounted in a virtual reality headset (Prime, Homido, France), controlled through a handheld Bluetooth remote control. The device weighs $465 \mathrm{~g}$. The smartphone has a total screen resolution of $2960 \times 1440$ pixels (approximately half width used per eye) and presents a digital image on an AMOLED screen. The headset's lenses provide an approximately 110 degree diagonal ( 80 degree horizontal) field of view, while the software offers approximately $0.7 x$ to $24.3 x$ magnification (38x relative zoom). Brightness, exposure, blue light filter and inter-pupillary distance are customisable. SightPlus has five image enhancement modes (Figure 2), each of which modifies the whole image: 'Normal mode' shows a full colour video feed. 'Enhanced mode' enables subtle feature enhancement by modulating selected spatial frequency components, resulting in sharper edges and smoother colours. 'Contrast mode' offers strong edge enhancement. 'Inverted mode' displays an inverted greyscale image, rendering white as black and vice versa. 'Text mode' offers a binary image (default: yellow on black) based on edge detection and thresholding, making it most suitable for reading. 
Data collection was performed following a low vision assessment conducted as part of a routine low vision clinic assessment by the first author. As part of this initial assessment, baseline visual acuity was measured binocularly with best refractive correction on a retro illuminated distance logMAR chart (Lighthouse Series ETDRS chart, Precision Vision, Woodstock, IL, USA). Letter-by-letter scoring was used and participants were encouraged to guess until no letters were correctly identified on a line. Where no letters were read at $4 \mathrm{~m}$, the chart was moved to 2 metres, 1 metre, and $50 \mathrm{~cm}$. If no letters were identified from $50 \mathrm{~cm}$, hand movements and perception of light was used. Contrast sensitivity was measured using a Pelli-Robson chart at 1 metre (Precision Vision, Woodstock, IL, USA) with the triplet being scored as read correctly when two of three letters were read. Reading performance (near visual acuity, critical print size, reading speed) was measured using the iPad version of the MNREAD test (Regents of the University of Minnesota, Minneapolis, MN, USA). ${ }^{21}$ Visual field data were extracted from the participant's medical records. Those with loss only within the central 15 degrees were classified as having central loss, those with loss only beyond 15 degrees as peripheral loss, those with loss in both regions as mixed, and without field loss as no field loss.

During the study session, each participant wore SightPlus for approximately 10-15 minutes under the supervision of the first author. Interpupillary distance of the device was adjusted optically and in software to ensure there was no diplopia and participants were allowed to become comfortable with its controls. All image enhancement modes were demonstrated. Once the participant was comfortable with the device and its controls, visual acuity, contrast sensitivity and reading performance were then assessed with the device: first in normal mode, and subsequently in the preferred image enhancement mode. Free choice of enhancement mode was allowed to explore the best-case scenario achievable with SightPlus; time constraints did not allow a systematic assessment of all modes. Participants were able to use a freely chosen zoom level for a given task. Data collection was terminated if participants self-reported nausea or any other unpleasant effects preventing them from continuing the session or if they expressed the wish to withdraw. 
Statistical analysis: efficacy

167 A repeated measures ANOVA was performed to compare between baseline, normal mode and preferred enhanced mode for distance visual acuity, contrast sensitivity and reading performance metrics (near visual acuity, critical print size and reading speed). In case of significance, pairwise post-hoc tests with Bonferroni correction were conducted.

Statistical analysis: willingness to use device

Willingness to use an ehLVA like SightPlus was assessed against the following 15 individual predictor variables through sub-group analysis: demographic factors (age, gender, work status (working, not working), current use of electronic magnifiers (yes, no)); disease factors (progressive or stable, time since diagnosis, percentage lifetime with sight loss); visual metrics (type of visual field loss (central, visual, peripheral, mixed, none) as well as baseline, difference and end point in normal mode for binocular visual acuity and binocular contrast sensitivity. For continuous variables, an independent ttest was performed, with 'would use' as the grouping variable. For nominal data, a $X^{2}$ test was performed. Stepwise multivariate regression was performed using the same demographic, disease and visual factors.

\section{Qualitative analysis}

At the end of the session, a semi-structured questionnaire was administered, where each participant was asked four of the following five questions:

1. 'Would you be willing to use a device like this?'

2. (If response to question 1 is 'yes'): 'what would you use it for?';

3. (If reponse to question 1 is 'no'): 'why not?'

4. 'What are the strengths of the device at present?' 
Participants were encouraged to answer each question as fully as possible. Key points were written,

192

193

194

195

196

197

and the investigator confirmed the responses with the participant. A grounded theory approach was used for analysis, where responses were coded and grouped into categories. Categories of responses are reported.

\section{Adverse effects}

Participants were asked whether they experienced nausea, claustrophobia, headache, eyestrain, or any other adverse response.

\section{Results}

Mean (SD) participant age was 51.4 (18.7) years. 23 participants (38\%) were female. Mean (SD) visual acuity with both eyes open was $0.82(0.39) \log M A R(6 / 40 ; 20 / 132)$ with a range of 0.04 $\log \operatorname{MAR}(6 / 7 ; 20 / 22)$ to hand movements. Eight participants $(13 \%)$ had no visual field loss, $27(45 \%)$ had central field loss, 17 (28\%) had peripheral field loss, and eight (13\%) had both central and peripheral field loss.

\section{Eleven participants had retinitis pigmentosa or Usher syndrome, 11 had other inherited retinal} diseases, 11 had inherited macular diseases, 6 had age-related macular disease, 6 had optic neuritis, 3 had albinism, 2 had glaucoma, and 2 had diabetic eye disease. One participant had each of optic atrophy, optic neuritis, congenital cataract, aniridia, achromatopsia, paediatric glaucoma, congenital stationary night blindness, chronic central serous retinopathy, and Leber's congenital amaurosis.

\section{A. Efficacy}

Visual acuity (Table 1, Figure 3 ) was significantly different between conditions $(F=223.14, p<$ $\left.0.0001, \eta_{p}^{2}=0.817\right)$. Post hoc analysis revealed a significant difference between baseline and normal mode $(p<0.0001)$ as well as baseline and preferred enhanced mode $(p<0.0001)$, but not between 
normal and enhanced mode $(p=0.292)$. Visual acuity improved by a mean (SD) of $0.63(0.34)$ $\log M A R$ - more than 6 lines on the sight chart - to $0.20(0.28) \log M A R$ in normal mode $(N=58)$ and by $0.70(0.32) \log M A R-7$ lines - to $0.16(0.26) \log M A R$ in preferred enhanced mode $(N=51)$. Visual acuity improved in all but one participant, achieving $0.2 \log$ MAR or better irrespective of baseline acuity in $67 \%$ of participants.

222

Contrast sensitivity (Table 1, Figure 3 ) was significantly different between conditions $(F=52.45, p<$ $\left.0.0001, \eta_{p}^{2}=0.527\right)$. Post hoc analysis revealed a significant difference between all pairwise comparisons $(p<0.0001)$. Contrast sensitivity improved by a mean (SD) of $0.22(0.30)$ log units to $1.21(0.47) \log$ units $(6 \%)$ in normal mode $(N=55)$ and by $0.40(0.31) \log$ units to $1.37(0.50) \log$ units (4\%) in preferred enhanced mode $(\mathrm{N}=48)$. 
243 (SD) of $24.98(30.55)$ words per minute to $56.02(37.30)$ words per minute in normal mode $(\mathrm{N}=43)$

244 and by 24.07 (34.16) words per minute to 62.23 (40.56) words per minute in preferred enhanced

245 mode $(\mathrm{N}=30)$.

246

247 Table 1. Efficacy metrics.

248 Outcomes for five efficacy metrics across the three study conditions. Conditions with the same superscript $(a, b)$ were significantly different from each other in pairwise post-hoc tests (Bonferroni correction) following a repeated measures ANOVA. Not all participants completed all conditions: for example, some participants were unable to align the device for certain tests, and some participants were unable to read with it. Where participants disliked all enhanced modes, assessment was not performed with the enhanced mode.

\begin{tabular}{|l|c|c|c|c|c|c|}
\hline & \multicolumn{3}{|c|}{ No device, baseline } & \multicolumn{2}{|c|}{$\begin{array}{c}\text { With device, normal } \\
\text { mode }\end{array}$} & \multicolumn{2}{|c|}{$\begin{array}{c}\text { With device, } \\
\text { preferred enhanced } \\
\text { mode }\end{array}$} \\
\hline & $\begin{array}{c}\text { Mean } \\
\text { (SD) }\end{array}$ & $\mathrm{N}$ & $\begin{array}{c}\text { Mean } \\
(\text { SD) }\end{array}$ & $\mathrm{N}$ & $\begin{array}{c}\text { Mean } \\
(\text { SD) }\end{array}$ & $\mathrm{N}$ \\
\hline $\begin{array}{l}\text { Distance Visual Acuity } \\
\text { (ETDRS, in logMAR) }\end{array}$ & $\begin{array}{c}0.82 \\
(0.39)^{\mathrm{a}, \mathrm{b}}\end{array}$ & 59 & $\begin{array}{c}0.20 \\
(0.28)^{\mathrm{a}}\end{array}$ & 59 & $\begin{array}{c}0.16 \\
(0.26)^{\mathrm{b}}\end{array}$ & 52 \\
\hline $\begin{array}{l}\text { Contrast sensitivity (Pelli- } \\
\text { Robson, in log units) }\end{array}$ & $\begin{array}{c}0.99 \\
(0.45)^{\mathrm{a}}\end{array}$ & 57 & $\begin{array}{c}1.21 \\
(0.47)^{\mathrm{a}}\end{array}$ & 56 & $\begin{array}{c}1.37 \\
(0.50)^{\mathrm{a}}\end{array}$ & 49 \\
\hline $\begin{array}{l}\text { Near Visual Acuity } \\
\text { (MNREAD, in logMAR) }\end{array}$ & $\begin{array}{c}0.56 \\
(0.30)\end{array}$ & 57 & $\begin{array}{c}0.44 \\
(0.32)\end{array}$ & 49 & $\begin{array}{c}0.46 \\
(0.31)\end{array}$ & 36 \\
\hline $\begin{array}{l}\text { Critical Print Size } \\
\text { (MNREAD, in logMAR) }\end{array}$ & $\begin{array}{c}0.75 \\
(0.24)\end{array}$ & 53 & $\begin{array}{c}0.54 \\
(0.33)\end{array}$ & 44 & $\begin{array}{c}0.55 \\
(0.33)\end{array}$ & 31 \\
\hline $\begin{array}{l}\text { Reading Speed (MNREAD, } \\
\text { in words/minute) }\end{array}$ & $\begin{array}{c}79.34 \\
(47.82)^{\mathrm{a}, \mathrm{b}}\end{array}$ & 53 & $\begin{array}{c}56.02 \\
(37.30)^{\mathrm{a}}\end{array}$ & 44 & $\begin{array}{c}62.23 \\
(40.56)^{\mathrm{b}}\end{array}$ & 31 \\
\hline
\end{tabular}



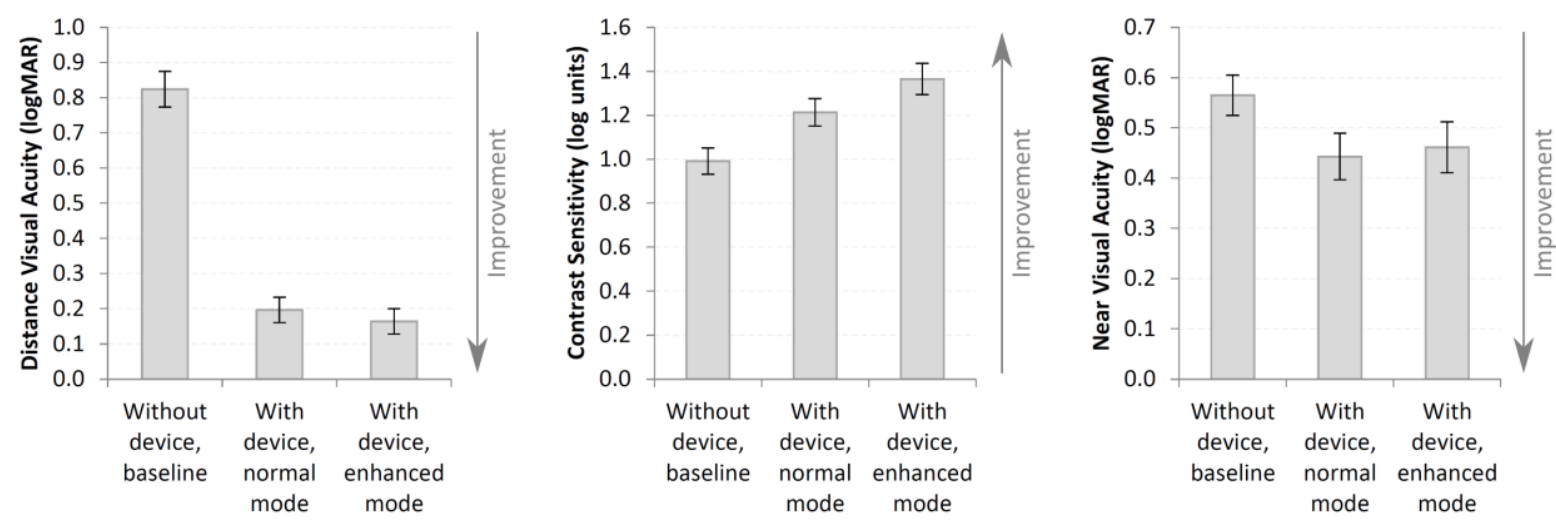

Figure 3. Mean (SE) distance visual acuity, contrast sensitivity and near visual acuity across the three

study conditions. 'Enhanced mode' describes the preferred enhanced mode chosen by participants.

260

\section{B. Willingness to use ehLVA}

A total of 28 participants (47\%) indicated that they would use an ehLVA like SightPlus, 27 participants (45\%) would not and 5 participants (8\%) were not sure.

Examined individually, seven variables had a significant effect on willingness to use an ehLVA like

SightPlus (Table 2): age, affected proportion lifetime, baseline contrast sensitivity, difference in visual acuity with SightPlus (baseline vs. normal mode), history (acquired vs. inherited), use of e-LVAs including smartphones/tablets (yes / no) and work status (working / not working).

Multivariate logistic regression resulted in a model with three variables: proportion of lifetime affected $\left(X^{2}=6.4, p=0.012\right)$, baseline contrast sensitivity $\left(X^{2}=5.6, p=0.018\right)$ and use of e-LVAs $\left(X^{2}=\right.$ 13.1, $p<0.001$ ). This model explained $41 \%$ of the observed variation (Cox and Snell pseudo $R^{2}=$ $0.405)$.

Table 2. Willingness to use ehLVA vs. individual variables.

275 Predictive power of individual variables for willingness to use an ehLVA like SightPlus. Outcomes of independent $\mathrm{t}$-tests for continuous variables and $\mathrm{X}^{2}$ tests for categorical variables. Effect size 


\begin{tabular}{|c|c|c|c|c|c|c|}
\hline & \multicolumn{4}{|c|}{ Would you use an ehLVA like SightPlus? } & \multirow[b]{3}{*}{$\begin{array}{c}\text { Effect size } \\
\text { (mean } \\
\text { difference) }\end{array}$} & \multirow[b]{3}{*}{ p-value } \\
\hline & \multicolumn{2}{|c|}{ No } & \multicolumn{2}{|c|}{ Yes } & & \\
\hline Variable & $\mathbf{N}$ & $\begin{array}{l}\text { Mean } \\
\text { (SD) }\end{array}$ & $\mathbf{N}$ & $\begin{array}{l}\text { Mean } \\
\text { (SD) }\end{array}$ & & \\
\hline Age (years) & 27 & $58(20)$ & 28 & $43(16)$ & 15 & $0.003^{*}$ \\
\hline $\begin{array}{l}\text { Time since diagnosis } \\
\text { (years) }\end{array}$ & 27 & $15(18)$ & 28 & $23(13)$ & -7 & 0.082 \\
\hline $\begin{array}{l}\text { Proportion lifetime with } \\
\text { sight loss (fraction, } 0 \text { to } 1 \text { ) }\end{array}$ & 27 & $\begin{array}{c}0.30 \\
(0.35)\end{array}$ & 28 & $\begin{array}{c}0.58 \\
(0.31)\end{array}$ & -0.28 & $0.003^{*}$ \\
\hline $\begin{array}{l}\text { Binocular VA, baseline } \\
\text { (logMAR) }\end{array}$ & 27 & $\begin{array}{c}0.72 \\
(0.42)\end{array}$ & 27 & $\begin{array}{c}0.89 \\
(0.35)\end{array}$ & -0.17 & 0.113 \\
\hline $\begin{array}{l}\text { Binocular CS, baseline (log } \\
\text { units) }\end{array}$ & 25 & $\begin{array}{c}1.18 \\
(0.35)\end{array}$ & 27 & $\begin{array}{c}0.92 \\
(0.46)\end{array}$ & 0.25 & $0.031^{*}$ \\
\hline $\begin{array}{l}\text { Binocular VA, end point in } \\
\text { normal mode (logMAR) }\end{array}$ & 26 & $\begin{array}{c}0.20 \\
(0.31)\end{array}$ & 28 & $\begin{array}{c}0.15 \\
(0.23)\end{array}$ & 0.05 & 0.476 \\
\hline $\begin{array}{l}\text { Binocular CS, end point in } \\
\text { normal mode (log units) }\end{array}$ & 23 & $\begin{array}{c}1.39 \\
(0.43) \\
\end{array}$ & 28 & $\begin{array}{c}1.19 \\
(0.42) \\
\end{array}$ & 0.19 & 0.108 \\
\hline $\begin{array}{l}\text { Binocular VA, difference in } \\
\text { normal mode (logMAR) }\end{array}$ & 26 & $\begin{array}{l}-0.53 \\
(0.33)\end{array}$ & 27 & $\begin{array}{l}-0.74 \\
(0.33)\end{array}$ & 0.20 & $0.029 *$ \\
\hline \multirow[t]{2}{*}{$\begin{array}{l}\text { Binocular CS, difference in } \\
\text { normal mode (log units) }\end{array}$} & 23 & $\begin{array}{c}0.19 \\
(0.30)\end{array}$ & 27 & $\begin{array}{c}0.28 \\
(0.30) \\
\end{array}$ & -0.09 & 0.278 \\
\hline & Count A & Count B & Count A & Count B & & \\
\hline $\begin{array}{l}\text { Gender (male }(A) / \text { female } \\
(B))\end{array}$ & 16 & 11 & 17 & 11 & & 1.000 \\
\hline $\begin{array}{l}\text { History (inherited }(A) / \\
\text { acquired }(B) \text { ) }\end{array}$ & 15 & 12 & 25 & 3 & & $0.007^{*}$ \\
\hline $\begin{array}{l}\text { Progression (progressive } \\
\text { (A)/ stable (B)) }\end{array}$ & 20 & 7 & 22 & 6 & & 0.758 \\
\hline $\begin{array}{l}\text { Use of e-LVAs (yes (A)/ no } \\
(B))\end{array}$ & 5 & 22 & 20 & 8 & & $<0.001^{*}$ \\
\hline $\begin{array}{l}\text { Work status (working (A)/ } \\
\text { not working (B)) } \\
\text { Visual field status (central, } \\
\text { full, mixed, peripheral) }\end{array}$ & & & & & & $\begin{array}{l}0.015^{*} \\
0.394\end{array}$ \\
\hline
\end{tabular}


281 A total of 35 participants (58\%) preferred the 'normal' mode, ten (17\%) preferred 'inverted', three

(5\%) preferred 'contrast', two preferred 'enhanced' and one preferred 'text'. The remaining seven participants reported that preference depended on the task.

D. Qualitative analysis

The 33 participants who indicated that they would use an ehLVA like SightPLus or that were not sure reported a total of 20 different activities as envisaged use cases: television $(N=14)$, reading $(N=7)$,

The most commonly cited strengths of the device were the image clarity $(\mathrm{N}=10)$, the image

brightness $(\mathrm{N}=6)$, the level of zoom $(\mathrm{N}=5)$ and its comfort $(\mathrm{N}=2)$.

The most frequent criticism of the device was its weight (too heavy, $\mathrm{N}=26$ ), aesthetic appearance the headset $(\mathrm{N}=5)$, not finding it helpful $(\mathrm{N}=4)$ and disorientation or problems with depth perception $(\mathrm{N}=4)$.

Adverse reactions

Most of the participants did not report any adverse reaction (48 of 60 participants, $80 \%$ ). Twelve participants (20\%) reported a single adverse reaction and none reported multiple adverse reactions. The most frequently reported adverse reactions were nausea $(N=4,7 \%)$ and dizziness $(N=4,7 \%)$. 
history of dry eye) described "sore eyes". No participants reported claustrophobia or eye strain. In all cases, symptoms resolved on removal of the headset while participants were in clinic. For six participants (three with nausea, and one with each of headache, sore eyes and dizziness), reactions were severe enough for the investigator to terminate the study session early. Data for these participants until the point of termination were analysed.

\section{Discussion}

\section{Summary of findings}

This study investigated improvements in vision and the willingness to use an ehLVA using SightPlus (GiveVision, UK). SightPlus provided clinically significant and functionally relevant improvements in visual acuity and contrast sensitivity: distance visual acuity improved by more than 6 lines on a $\operatorname{logMAR}$ chart, and contrast sensitivity improved by 8 letters with image enhancement. Half of our study population indicated willingness to use the device. Examining predictor variables individually, we found a significant effect for willingness to use an ehLVA for those with younger age, longer proportion of lifetime with visual impairment, lower baseline contrast sensitivity, greater improvement in visual acuity with SightPlus (baseline vs. normal mode), inherited sight loss (compared to acquired sight loss), existing use of electronic LVAs (including smartphones/tablets) and working. Multivariate logistic regression reduced this parameter space to the proportion of lifetime affected, baseline contrast sensitivity and use of electronic LVAs, explaining $41 \%$ of the variation in the data. This illustrates that while the examined factors may predict the likelihood of uptake to a degree, factors beyond the scope of this study also play a role. While we anticipated that current use of electronic LVAs would predict willingness to use, we were surprised to find that baseline acuity was not a predictor. This shows that lifestyle and other factors maybe more important for the relevance of ehLVAs. 
Improvements in performance on standard sight tests were similar to those reported for the eSight ehLVA device, which gave a mean (SD) of $0.20(0.31) \log$ MAR (compared to $0.20(0.28)$ logMAR in the present study) and contrast sensitivity of $1.44(0.44)$ log units (compared to $1.21(0.47)$ log units in normal mode and $1.37(0.50)$ log units with preferred enhanced mode in the present study). ${ }^{17}$ While participants in the eSight trial were self-selected through wanting to use eSight, participants in the present study were a random sample from a low vision clinic. Given the similarity between our results and the eSight study, a prior disposition of wanting to use an ehLVA is unlikely to predict efficacy.

For reading performance, we found a smaller positive effect compared to eSight: ${ }^{17}$ near visual acuity and critical print size improved significantly with SightPlus, albeit to a smaller degree (near visual acuity improved from 0.90 to $0.33 \log$ MAR with eSight and 0.56 to $0.44 \log$ MAR with SightPlus; critical print size improved from 1.08 to $0.59 \log M A R$ for eSight and 0.75 to $0.54 \log M A R$ with SightPlus). While in the eSight study there was no significant effect of the ehLVA on reading speed, ${ }^{17}$ we detected a significant decrease in reading speed with SightPlus (from 79 to 56 words per minute in normal mode and to 62 words per minute in preferred enhanced mode). A reduction in reading speed could be explained by reduced visual span, or by image movement. A 2018 review of studies evaluating reading aids for people with low vision found that reading speed may be highest in standmounted electronic CCTVs compared to optical devices, with further evidence for longer reading durations and better ease of use. ${ }^{22}$ Comparing stand-mounted CCTV and head-mounted devices (HMDs), the review reported similar performance between the two: for HMDs, on average 66 words per minute and near visual acuity of $0.92 \log M A R$; for CCTVs, 3.1 words per minute more for reading speed and $0.05 \operatorname{logMAR}$ more for near visual acuity. It is important to note that near visual acuity for the ehLVAs reported here substantially exceeded those outcomes. Despite the reduced speed in our study, several participants indicated that they would choose to use SightPlus for reading, perhaps 
because of the increased working distance, comprehension or comfort of reading, none of which we assessed.

Willingness to use

Half of our study population indicated that they were willing to use an ehLVA like SightPlus,

Similarly, a recent large-scale review into functional sight in AMD reported that face recognition, perception of scenes, computer use, meal preparation, shopping, cleaning, watching TV, reading and self-care are among the tasks negatively affected by sight loss. ${ }^{23}$ Future ehLVAs should aim to support such common activities to enable independence and improvement in quality of life.

Seven individual variables predicted a higher willingness to use an ehLVA like SightPlus. Our results agree with the literature summarised in a recent review: ${ }^{24}$ for example, younger age has been found to be a predictor of better compliance; duration of vision loss may predict greater uptake (similar to our 'proportion of lifetime affected' predictor). In our study, as in most previous work, there was no effect of baseline acuity on uptake or benefit. Our univariate and multivariate analysis showed that existing use of electronic devices was a strong predictor for willingness to use an ehLVA like SightPlus. It is not clear whether comfort with technology was a significant factor in this. The role of technological literacy should be explored in future studies, especially for older people. Proportion of lifetime affected and baseline contrast sensitivity also had an effect in this model. Those diagnosed for proportionally longer may have adapted more to their sight loss and be more willing to seek assistance, ${ }^{25}$ although this requires further research.

The most common reasons for not wanting to use the device were its weight, $465 \mathrm{~g}$ (26 of 60 participants), its appearance (14 of 60 participants) and perceived image movement or image lag (12 of 60 participants). Weight and appearance are inherent to virtual reality (VR) headset-based 
solutions like SightPlus or IRIS Vision, although can be overcome through bespoke designs such as that pioneered by eSight. In future, creating lighter and more comfortable to wear devices will be an important design consideration. Perceived image movement and image lag can arise from head movement, throttled processing speed or optical image stabilisation resulting in slight lag of the scene relative to head movement. Improved image stabilisation, zero-lag image processing and compensation for fast changes in head orientation could all make this effect less noticeable.

Exactly half of those who met the World Health Organisation criterion for low vision (visual acuity poorer than $6 / 18$ ) would use a ehLVA like SightPlus, compared to $47 \%$ of our complete sample. We could not determine the lower acuity limit for SightPlus to be useful: the participant with the poorest visual acuity (perception of light in the right eye, hand movements in the left eye) improved to $0.06 \log \operatorname{MAR}(6 / 7 ; 20 / 23)$ and would use the device for work and television.

\section{Outlook}

As more wearable low vision devices become available, a standardised test battery or unified task inventory of real-life activities would enable true comparison of different devices and quantify their impact on performance of common daily tasks. No such comparison has yet been performed, and limited data are available on new wearable devices. ${ }^{2}$ Assessment of visual function beyond acuity and contrast sensitivity has been called for in a large-scale review of visual function and quality of life in $A M D^{23}$ and would enable comprehensive evaluation and benchmarking of all types of low vision aids. A comparative study of devices would be invaluable to clinicians, patients and carers. Our clinical experience with this device suggests that existing inventories may need to be revised to reflect common, modern real-world tasks across a broad age group. Any such evaluation package should contain tasks most commonly needing visual aids by people with low vision while being repeatable across different institutions. Examples of this include the inclusion of face recognition by Wittich ${ }^{17}$ and a bespoke (albeit unvalidated) task inventory used by Orcam. ${ }^{18}$ Future work should 
expand on this to include measurable performance, avoiding the confounding effects of selfreporting and observation. The impact of ehLVAs on quality of life needs to be established in order to facilitate any future health economic assessments.

\section{Study limitations}

Our inclusion criteria were deliberately broad in order to determine who would most benefit from this system. Since we did not find clear cut-offs with regards to minimum or maximum baseline visual acuity for those who benefit from an ehLVA, future work should either use a stratified sampling method with a larger sample size, or focus on specific disease conditions to identify patient beneficiaries more robustly. Visual acuity may not be a predictor of benefit or uptake, and future work should continue to examine a broad range of predictor variables.

In this study, we allowed participants free choice of the image enhancement mode which they found most useful for a given task. Our results for 'preferred enhanced mode' hence contain different modes. Although visual acuity and contrast sensitivity improved further with image enhancement as well as zoom, $58 \%$ of participants preferred the 'normal' mode. It is important to note that the outcomes we used were letter and text based. Enhancement mode preferences for images containing a broader range of colours and spatial frequencies (such as natural scenes or TV) may differ. Previous research has shown that participants tend to prefer the most natural looking image rendering through lower level enhancements, and that preferences depend on the nature of sight loss and image content. ${ }^{1626}$ Further work is needed to link image enhancement modes to tasks and sight loss conditions, as has been attempted by other groups. ${ }^{12} 13$

Adverse reactions were reported by 12 participants (20\%), with nausea and dizziness being the most common unpleasant sensations. Nausea has been reported as a side-effect of wearable devices. ${ }^{817}$ 
to complete the full study session due to adverse effects. Future work should investigate the causes of these adverse effects and should endeavour to remove them by, for example, providing a less enclosed design.

\section{Conclusions}

This augmented reality low vision aid improves visual acuity, contrast sensitivity and near visual acuity. Approximately half of the people we demonstrated it to would want to use it, primarily for distance tasks. Uptake of the device would be higher if it was lighter and more cosmetically acceptable. We recommend that adults with low vision are given the opportunity to evaluate ehLVAs until larger scale studies can help better characterise those for whom these devices will prove particularly beneficial.

The efficacy of ehLVAs has now been demonstrated in two devices with different form factor and display technology (eSight and SightPlus). Research now needs to move beyond the clinic, to quantify the benefits of these systems for visually impaired people at work, in education, and at home. We hope that electronic, head-mounted low vision aids become more widely used and that they will increase the number of tasks which can be easily performed by people with visual impairment.

\section{References}

1. Moshtael H, Aslam T, Underwood I, Dhillon B. High Tech Aids Low Vision: A Review of Image

2. Deemer AD, Bradley CK, Ross NC, Natale DM, Itthipanichpong R, Werblin FS, et al. Low Vision Enhancement with Head-mounted Video Display Systems: Are We There Yet? . Optom Vis Sci 2018;95(9):774-84.

3. Ehrlich J, Ojeda L, Wicker D, Day S, Howson A, Lakshminarayanan V, et al. Head-Mounted Display Technology for Low-Vision Rehabilitation and VisionEnhancement. Am J Ophthalmol 2017;176:26-32. 
4. Massof RW, Rickman DL. Obstacles encountered in the development of the low vision enhancement system. Optom Vis Sci 1992;69(1):32-41.

5. Itoh Y, Klinker G. Vision enhancement: defocus correction via optical see-through head-mounted displays. Proc. 6th Augment. Hum. Int. Conf 2015:1-8.

6. Zhao Y. ForeSee : A Customizable Head-Mounted Vision Enhancement System for People with Low Vision. ASSETS '15 17th Int. ACM SIGACCESS Conf. Comput. Access 2015:239-49.

7. Moshtael H, Fu L, Underwood I, Dhillon B. Evaluation of Head-Mounted Displays for Macular Degeneration: A Pilot Study. In: Chen YW, Tanaka S, Howlett R, Jain L, editors. Innovation in Medicine and Healthcare 2016. InMed 2016. Smart Innovation, Systems and Technologies, Vol 60. Cham, Switzerland: Springer, 2016.

8. Deemer A, Deremeik J, Massof RW, Bradley C, Fujiwara K, Werblin FS, et al. Evaluation of a Virtual Bioptic Telescope and Virtual Projection Screen for Low Vision Patients. Invest Ophthalmol Vis Sci 2018;59(9):2563.

9. Hwang AD, Peli E. An augmented-reality edge enhancement application for Google glass. Optom Vis Sci 2014;91(8):1021-30.

10. Lawton T. Improved word recognition for observers with age-related maculopathies using compensation filters. Clin Vis Sci 1988;3(2):125-35.

11. Lawton TB. Improved Reading Performance Using Individualized Compensation Filters for Observers with Losses in Central Vision. Ophthalmology 1989;96(1):115-26.

12. Leat SJ, Mei M. Custom-devised and generic digital enhancement of images for people with maculopathy. Ophthalmic \& physiological optics 2009;29(4):397-415.

13. Leat SJ, Omoruyi G, Kennedy A, Jernigan E. Generic and customised digital image enhancement filters for the visually impaired. Vision Res 2005;45(15):1991-2007.

14. Peli E, Goldstein RB, Young GM, C.L. T, Buzney SM. Image enhancement for the visually impaired. Simulations and experimental results. Invest Ophthalmol Vis Sci 1991;32(8):2337-50.

15. Peli E, Lee $\mathrm{E}$, Trempe CL, Buzney S. Image enhancement for the visually impaired: the effects of enhancement on face recognition. J Opt Soc Am A 1994;11(7):1929-39.

16. Tang J, Kim J, Peli E. Image enhancement in the JPEG domain for people with vision impairment. IEEE transactions on biomedical engineering 2004;51(11):2013-23.

17. Wittich W, Lorenzini M-C, Markowitz SN, Tolentino M, Gartner SA, Goldstein JE, et al. The Effect of a Head-mounted Low Vision Device on Visual Function. Optom Vis Sci 2018;95(9):774-84.

18. Moisseiev E, Mannis M. Evaluation of a Portable Artificial Vision Device Among Patients With Low Vision. JAMA Ophthalmol 2016;134(7):748-52.

19. Crossland MD, Silver J. Thirty years in an urban low vision clinic: Changes in prescribing habits of low vision practitioners. Optometry and vision science 2005;82:617-22.

20. Leat SJ, Legge GE, Bullimore MA. What is low vision? A re-evaluation of definitions. Optom Vis Sci 1999;76(4):198-211.

21. Calabrèse $A$, To L, Berkholtz E, Rafian P, Legge GE. Comparing performance on the MNREAD iPad application with the MNREAD acuity chart. Journal of vision 2018;18(1):8.

22. Virgili G, Acosta R, Grover LL, Bentley S, Giacomelli G. Reading aids for adults with low vision. Cochrane Database Syst Rev 2013;23(10):CD003303.

23. Taylor DJ, Hobby AE, Binns AM, D.P. C. How does age-related macular degeneration affect realworld visual ability and quality of life? A systematic review. BMJ Open 2016;6(12):e011504.

24. Lorenzini $M-C$, Wittich $W$. Factors related to teh use of magnifying low vision aids: a scoping review. Disabil Rehabil 2019;23:1-13.

25. Crossland MD, Culham LE. Psychological aspects of visual impairment. Optometry in Practice 2000;1(1):21-26.

26. Fullerton M, Peli E. Digital Enhancement of Television Signals for People with Visual Impairments: Evaluation of a Consumer Product. J. Soc. Inf. Disp. 2008;16(3):493-500. 